Revue d'histoire de l'Amérique française

DAU REVEE D.HISTOIRE DE L'AMÉRIQUE FRANÇAISE

\title{
Les travailleurs à la construction du chemin de fer dans la région de Sherbrooke (1851-1853)
}

\section{Jean-Pierre Kesteman}

Volume 31, numéro 4, mars 1978

URI : https://id.erudit.org/iderudit/303650ar

DOI : https://doi.org/10.7202/303650ar

Aller au sommaire du numéro

Éditeur(s)

Institut d'histoire de l'Amérique française

ISSN

0035-2357 (imprimé)

1492-1383 (numérique)

Découvrir la revue

Citer cet article

Kesteman, J.-P. (1978). Les travailleurs à la construction du chemin de fer dans la région de Sherbrooke (1851-1853). Revue d'histoire de l'Amérique française, 31(4), 525-545. https://doi.org/10.7202/303650ar d'utilisation que vous pouvez consulter en ligne. 


\title{
LES TRAVAILLEURS À LA CONSTRUCTION DU CHEMIN DE FER DANS LA RÉGION DE SHERBROOKE (1851-1853)*
}

\author{
Jean-Pierre Kesteman \\ Département d'bistoire \\ Université de Sherbrooke
}

La construction, de 1845 à 1853 , du chemin de fer St. Lawrence and Atlantic, destiné à ouvrir à Montréal la porte océanique de Portland (Maine), et intégré bientôt dans le réseau du Grand-Tronc, passe souvent pour le premier chapitre de l'épopée ferroviaire canadienne ${ }^{1}$. Comme pour d'autres entreprises de chemin de fer au Canada, les historiens du St. Lawrence and Atlantic se sont surtout attachés à relater les difficultés rencontrées par les promoteurs, tant pour se procurer les capitaux nécessaires que pour braver une nature souvent hostile. De l'historiographie de cette compagnie ferroviaire se dégage donc une vision triomphaliste à la gloire des hommes politiques, des actionnaires et des ingénieurs qui y ont œuvré $^{2}$. La personnalité d'Alexander T. Galt, qui en fut un des directeurs et actionnaires

* Une première version de cet article a fait l'objet d'une communication présentée à l'Université du Québec à Rimouski, le 30 septembre 1977, au Congrès de l'Institut d'histoire de l'Amérique française.

1 L'histoire de cette compagnie ferroviaire est exposée dans A.W. CURRIE, The Grand Trunk Railway of Canada (Toronto, Un. of Toronto Press, 1957), 556 p., ainsi que dans G.R. STEVENS, History of the Canadian National Railway (New York, Macmillan, 1973), 538 p., Id., Canadian National Railways, vol. 1 - Sixty Years of Trial and Error (1836-1896) (Toronto, Clarke et Irwin, 1960), 514 p., p. $52-83$ (cité Sixty years)

2 A commencer par J.M. et E. TROUT, The Railways of Canada (Toronto, 1871; Coles reprint, Toronto, 1970), 52 et 57-86. Voir aussi O.D. SKELTON, The Railway Builders (Toronto, Glasgow and Brook, 1916), 40-79; N. THOMPSON et J.H. EDGAR, Canadian Railway Development form the Éarliest Times (Toronto, Macmillan, 1933), 11-16; J.J. LANNING, «Historical Sketch of the Grand Trunk Railway " in Canada. An Encyclopedia of the Country, J.C. HOPKINS, ed., (6 vols, Toronto, 1898-1900), 2: 104-111.

RHAF, vol. $31, \mathrm{n}^{\circ} 4$ (mars 1978) 
principaux, a été particulièrement célébrée ${ }^{3}$, sans que soient oubliés des constructeurs comme Thomas Brassey ${ }^{4}$ ou des ingénieurs comme Casimir Gzowski ${ }^{5}$.

Dès les années 1860 cependant, une commission parlementaire avait commencé à lever le voile sur les manipulations politiques, les conflits d'intérêts, les profits éhontés et la piètre qualité technique de l'entreprise ${ }^{6}$. Quelques études, utilisant le rapport de cette commission, donnent ainsi de l'histoire du St. Lawrence and Atlantic et du Grand-Tronc une version beaucoup plus critique ${ }^{7}$.

De tous ces écrits, les travailleurs qui ont œuvré à la construction de la ligne de Montréal à Portland sont les grands absents; cette lacune, commune à l'histoire de nombreuses entreprises canadiennes au $\mathrm{XIX}^{\mathrm{e}}$ siècle, n'est guère surprenante dans le cas des chemins de fer. Travailleur migrant, suivant au cours des mois et des années la progression de la voie, l'ouvrier constructeur de chemins de fer a laissé dans les documents encore moins de traces que ses homologues des manufactures urbaines. Sa condition de nomade l'a exclus des institutions municipales ou religieuses qui permettraient de mieux le connaître. D'autre part, comme les compagnies ferroviaires pratiquaient généralement le système des sous-contrats, les équipes de travailleurs n'étaient pas officiellement au service des compagnies et les rapports ou archives de celles-ci, quand elles existent, sont muettes pour tout ce qui concerne les bâtisseurs de la voie ferrée.

Les journaux des régions traversées par le chemin de fer jettent bien quelque lumière sur les aspects les plus sensationnels de l'activité des chantiers, comme les accidents de travail, les bagarres, l'alcoolisme, voire les grèves ou les émeutes. Ces sources locales sont, malgré leur intérêt, rarement impartiales: le travailleur du chemin de fer y est en effet reçu comme un fauteur de troubles, tout 1920).

3 O.D. SKELTON, Life and Times of Sir Alexander Tilloch Galt (Toronto,

4 A. HELPS, Life and Labours of Mr. Brassey, 1805-1870 (Londres, Bell and Daldy, 1872), 183-213.

5 L. KOS-RABCEWICZ-ZUBROWSKI et W.E. GREENING, Sir Casimir Stanislaus Gzowski. A Biography (Toronto, Burns and MacEachern, 1959), 213 p. (cité Gzowski).

6 Leg. Council Sessional Papers, vol. 13 (1854-1855): app. AAAA.

7 T.S. BROWN, $A$ History of the Grand Trunk Railway of Canada (Quebec, Hunter and Rose, 1864); G. MYERS, A History of Canadian Wealth (Chicago, Kerr, 1914), 179, 181, 208. 
juste bon à s'enivrer ou à molester «les honnêtes citoyens». Chaque ville subit ce mal nécessaire, puisqu'il est gage de la prospérité, mais souhaite voir déguerpir au plus tôt ces encombrants visiteurs.

Cette catégorie de travailleurs aurait donc pu laisser fort peu de traces si nous n'en avions pas retrouvé un nombre élevé, mentionné dans les recensements nominaux de 1851-1852 du comté de Sherbrooke. De fait, en octobre 1851, la ligne du St. Lawrence and Atlantic avait été ouverte de Longueuil à Richmond et les travaux de construction se poursuivaient vers Sherbrooke et la frontière américaine; de nombreux chantiers étaient occupés par des équipes de travailleurs sur une distance de $\mathbf{3 0}$ milles entre Richmond et Lennoxville. C'est là que les recenseurs les ont répertoriés entre la fin de janvier et la mi-mars 1852 , dans six unités de recensement du comté de Sherbrooke, soit Melbourne, Shipton (Richmond), Windsor, Brompton, Orford et Ascot (Sherbrooke) ${ }^{8}$. Bien qu'éparpillées parmi la population sédentaire, environ 1,800 personnes liées à l'activité des chantiers ferroviaires apparaissent ainsi dans un document de nature statistique, le plus ancien du genre, à notre connaissance, sur ce type de travailleurs au Canada.

Jointes aux articles de journaux de la région ${ }^{9}$ ou à des témoignages d'ingénieurs de l'époque ${ }^{10}$, ces données du recensement constituent la source principale de cette étude sur les caractéristiques sociales et les conditions de vie des travailleurs ferroviaires; quelques questions de méthode à propos de ces sources requièrent toutefois certaines précisions préliminaires.

\section{Questions méthodologiques}

Le premier problème consiste à repérer dans la masse de la population recensée quelles sont les personnes travaillant ou vivant dans les chantiers de construction des chemins de fer. La vallée de la rivière Saint-François, qu'empruntait le tracé de la voie, était en 1852 une zone presque entièrement défrichée et les villages de Richmond, Melbourne, Windsor, Lennoxville ainsi que la ville de

8 Recensements du Canada (1851), cahiers manuscrits microfilmés, nos C 1142-1143 (cités RC).

9 Sherbrooke Gazette (1851-1853); Stanstead Journal (1851-1853).

10 A. HELPS, op. cit.; Daylight through the Mountain. Letters and Labours of Civil Engineers Walter and Francis Shanly, F.N. WALKER, ed., (Toronto, Engin. Inst. of Canada, 1957), 442 p. (cité Shanly); Gzowski, op. cit. 
Sherbrooke constituaient déjà des points de concentration humaine relativement importants ${ }^{11}$. Les chantiers s'insèrent donc dans une région qui compte plusieurs milliers d'habitants. Relativement aisés à discerner dans les recensements nominaux pour les secteurs ruraux, les travailleurs des chantiers ferroviaires le sont un peu moins en zone urbaine comme à Sherbrooke, par exemple.

Bien que n'utilisant pas tous les mêmes méthodes, les officiers recenseurs ont laissé sur les pages de leurs documents suffisamment d'indices pour que l'historien puisse aujourd'hui retracer, sans grande marge d'erreur, les travailleurs dont nous parlons.

1. La colonne [22] des recensements nominaux (profession ou occupation) spécifie souvent le lien avec le chemin de fer (Railroad laborer, Railroad contractor).

2. Quelquefois l'occupation mentionnée est simplement laborer. Certes, ce mot désigne aussi bien les journaliers que les ouvriers non-spécialisés des villes. Le risque de confusion est cependant pratiquement écarté car le recenseūi ajoüute soüvent en marge, des indications permettant de repérer les journaliers ferroviaires $^{12}$.

3. La colonne [31] des recensements nominaux (type d'habitation) comporte, à côté des maisons de brique, en charpente de bois ou en bois rond, communes dans la région, des séries de "camps" (shanties) habités par des laborers ou Railroad laborers. Ici encore, les indications marginales des recenseurs ne laissent aucune ambiguité sur le type de chantiers: ce sont des chantiers ferroviaires et non, par exemple, des chantiers forestiers ${ }^{13}$.

Les indications manuscrites des recenseurs permettent donc de retracer la plupart des travailleurs œuvrant à la voie. Dans la zone urbaine de Sherbrooke, le nombre d'artisans et de journaliers recensés dépasse le chiffre de 1,200 personnes; cependant, tous ne sont pas liés à l'activité ferroviaire proprement dite. Sherbrooke entre dans l'ère de la vapeur et la fièvre de construction domiciliaire ou industrielle qui accompagne cette importante mutation, attire plu-

11 Voir infra, Tableau I.

12 Exemple: «this sheet contains nothing but Railway labourers who live in log shantys (sic)» (RC, Melbourne, 4).

13 Exemples: "all those persons residing in shanties are not permanent inhabitants but merely labourers on the Railway" (RC' Sherbrooke in Ascot, 104); «shanties occupied by Railroad labourers» (RC, Shipton, 124). 
sieurs centaines d'artisans du bâtiment, du charroi, de l'alimentation ${ }^{14}$. Parmi eux, un nombre appréciable, bien qu'indéterminé, doit travailler à la périphérie de la construction de la voie, comme par exemple à bâtir la gare ou l'atelier de locomotives. Ils demeurent, comme d'autres ouvriers de Sherbrooke, éparpillés dans les trois quartiers de la ville, dans des logements généralement surpeuplés ${ }^{15}$. Ils n'apparaissent pas de ce fait dans les cahiers du recenseur comme une catégorie liée au chemin de fer et ne peuvent donc être pris en considération ici. Par contre, le groupe de travailleurs directement liés aux travaux d'établissement de la voie, tels le dynamitage, le terrassement, le ballast, la pose des voies, la construction des ponts, vivent près du tracé de la ligne, généralement dans des "camps", même à proximité de la ville. Ils sont dès lors répertoriés à part par le recenseur et comme tels bien identifiables; seul ce dernier groupe, au moins pour l'unité de recensement de Sherbrooke (Ascot), sera compris dans l'analyse pratiquée pour cet article.

Il faut relever pour terminer quelques cas où le recenseur ne nous a pas livré de recensement nominal. Dans la division de Windsor, le recenseur Jesse Bartlett ne fournit pas de renseignements précis pour 215 des 297 personnes vivant dans les chantiers. Ses explications, mentionnées en marge, illustrent d'ailleurs bien le monde mouvementé des chantiers. La première zone incomplètement recensée touche un chantier de 75 Canadiens français, que, faute d'interprète, le recenseur n'a pu, dit-il, interroger ${ }^{16}$. Deux autres zones dans Windsor n'ont pu être mentionnées pour des questions de sécurité personnelle; c'est en tout cas ce que Bartlett en a déduit des explications de l'entrepreneur ${ }^{17}$. Dans la région de RichmondSherbrooke, c'est le seul secteur mal recensé, mais il ne soustrait à l'analyse qu'environ $12 \%$ de l'ensemble des 1857 personnes vivant dans les chantiers.

14 La population de Sherbrooke passe de 600 habitants en 1844 à 1900 en 1851. Cette transformation de la ville est décrite par un témoin oculaire dans $\mathrm{J}$. VANSITTART, Lifelines. The Stacey Letters 1836-1858 (Londres, 1976), 123, 126, 131, 138.

15 RC, Sherbrooke dans Orford; Sherbrooke dans Ascot.

16 "The shanty mention on line 25 built by Abram Dion contractor contains about 75 or 87 (?) labourers on Railroad. Their names, ages and residences not know correctly. Neither was I able to obtain an interpretor that I might be better informed. " (RC, Windsor, 15).

17 "The reason why I did not a certain (sic) the names of the labourers on these 2 sections was the contractor informed me if I went in with (...) asking questions, they could not answer for my personal safety». (RC, Windsor, 24). 


\section{Étude sociologique des chantiers}

Par leur nature même, les recensements nominaux nous permettent d'établir des statistiques sur divers aspects liés à l'état civil des personnes vivant dans les chantiers: population totale, statut familial, âge, lieu d'origine, religion, profession. Ils nous révèlent également des données qui éclairent le type et la qualité de vie de ces lieux de travail: catégories et densité d'habitation, fréquentation scolaire, présence d'animaux, statut de pensionnaire ou d'occupant principal d'un logement. Malgré leur aridité, ces statistiques recréent ainsi pour l'historien le décor complexe des chantiers ferroviaires.

\section{1- Population totale des chantiers}

En lisant les journaux régionaux du temps, on ne peut manquer d'être frappé par l'émoi causé dans la population sédentaire par la présence des ouvriers du chemin de fer, voire même la terreur ressentie ${ }^{18}$. Les recensements permettent de donner à ces sentiments une base quantitative et une explication objective. Le tableau I évoque l'ampleur du bouleversement démographique opéré par la présence des ouvriers des chantiers.

Pour les six unités de recensement de la zone RichmondSherbrooke, on peut ainsi compter, durant l'hiver de 1852, plus de 1800 personnes vivant dans les chantiers. Cette population constitue dans les zones proches de la voie ferrée une part importante de la population totale, surtout dans les cantons ruraux. Ainsi, Windsor et Brompton comptent presque autant de personnes dans les chantiers ferroviaires que dans les fermes de tout le canton. Si le pourcentage est plus faible dans les autres unités de recensement, c'est que ces unités correspondent à des cantons largement défrichés et peuplés sur toute leur étendue. Concentrée le long du tracé de la voie et non pas éparpillés dans la campagne comme les cultivateurs, les chantiers créent sur les chemins voisins de la ligne, surtout en zone rurale, des concentrations humaines qui dépassent largement les populations sédentaires. Pour l'ensemble de la région, la population des chantiers représente $19 \%$ de la population totale et à Sherbrooke, ce chiffre atteint $26 \%$. La présence de ces importants contingents a donc bien effectivement joué d'un grand poids dans la vie quotidienne des régions traversées.

18 Sherbrooke Gazette, 2 août 1851. 
TABLEAU I

Population des chantiers et population sédentaire (1852)

\begin{tabular}{|c|c|c|c|c|c|c|c|c|}
\hline no & & Shipton & Melbourne & Windsor & Brompton & Orford & Sherbrooke & TOTAL \\
\hline 1 & $\begin{array}{l}\text { nombre de person- } \\
\text { recensées pour } \\
\text { la construction du } \\
\text { chemin de fer }\end{array}$ & 307 & 109 & 297 & 360 & 81 & 703 & 1857 \\
\hline 2 & $\begin{array}{l}\text { population totale } \\
\text { du township selon } \\
\text { le recensement de } \\
1851 \text { (y compris } 1)\end{array}$ & 3285 & 2016 & 590 & 749 & 581 & 2557 & 9778 \\
\hline 3 & $\begin{array}{l}\% \text { de } 1 \text { par rapport } \\
\text { à } 2\end{array}$ & 9 & 5 & 50 & 48 & 14 & 27 & 19 \\
\hline 4 & $\begin{array}{l}\text { population «séden- } \\
\text { taire» }\end{array}$ & 2978 & 1907 & 293 & 389 & 500 & 1854 & 7921 \\
\hline
\end{tabular}




\section{2- Statut familial}

Contrairement à une image assez répandue, qui associe aux chantiers des hommes célibataires ou éloignés de leur famille, ce qui frappe dans la construction du chemin de fer en 1852, c'est l'importance des cellules familiales (Tableau II).

Sur les 1642 personnes pour lesquelles nous disposons de toutes les données, 987 (soit 60\%) sont rattachées à des structures familiales présentes sur le chantier. On dénombre 217 ménages, comptant 499 enfants «à charge » et 54 qui travaillent déjà au chantier. Image imprévue qui ressort de cette statistique, celle d'enfants d'âges divers (30\% de la population totale) au milieu de l'activité du chantier.

Les familles sont relativement de jeunes ménages, même lorsque les conjoints sont dans la trentaine, et la moyenne d'enfants par ménage est entre 2 et 3 (exactement 2.54). Un calcul mené sur les 269 enfants de l'unité de Sherbrooke donne une moyenne d'âge de 5.69 ans.

La famille constitue d'ailleurs l'épine dorsale des chantiers, car elle correspond généralement à l'unité d'habitation ; on retrouve habituellement un ménage par «camp», plus rarement deux. Par ailleurs, c'est à ces familles que l'autre partie de la population des chantiers, célibataires en majorité, sont en général intégrés. La plupart des camps hébergent en effet en qualité de pensionnaires un nombre variable de ces travailleurs (en moyenne 2.55 par ménage). Il faut y ajouter des hommes mariés, parfois accompagnés de leurs fils aînés, qui ont laissé leur famille dans une autre ville.

\section{3- Âge des travailleurs}

Le recensement signalant les âges de chaque individu recensé, il est possible d'établir une statistique précise dans ce domaine. On ne s'étonnera pas d'y voir des enfants de 10 ans et des vieux travailleurs de 70 ans. Le tableau III permet de voir la jeunesse relative de la main-d'œuvre non spécialisée: près de la moitié $(45 \%)$ a moins de 25 ans et les travailleurs de plus de 35 ans ne constituent qu'un quart du contingent. Des calculs par secteurs de recensement indiquent que c'est à Sherbrooke, donc à la pointe de l'avancée de la construction de la ligne, que se trouve le plus grand nombre de jeunes travailleurs ( $51 \%$ ont moins de 25 ans contre $42 \%$ dans les cinq autres secteurs). 
TABLEAU II

Logement et condition familiales des travailleurs des chantiers

\begin{tabular}{|c|c|c|c|c|c|c|c|c|}
\hline no & & Melbourne & Shipton & Windsor & Brompton & Orford & Sherbrooke & TOTAL \\
\hline \multirow[t]{2}{*}{1} & $\begin{array}{l}\text { nombre total d'habitations } \\
\text { comprenant: }\end{array}$ & 15 & 44 & 27 & 41 & 8 & 70 & 205 \\
\hline & $\begin{array}{l}\text { a) camps ( shanties) } \\
\text { b) } \log \text { cabins } \\
\text { c) maisons de bois } \\
\text { d) maisons de brique }\end{array}$ & $\begin{array}{r}14 \\
1 \\
-\end{array}$ & $\begin{array}{r}34 \\
1 \\
5 \\
4\end{array}$ & $\frac{23}{6}$ & $\begin{array}{r}38 \\
2 \\
1 \\
-\end{array}$ & $\begin{array}{l}5 \\
1 \\
2 \\
-\end{array}$ & $\begin{array}{r}59 \\
10 \\
1\end{array}$ & $\begin{array}{r}171 \\
5 \\
24 \\
5\end{array}$ \\
\hline 2 & $\begin{array}{l}\text { nombre total de } \\
\text { personnes }\end{array}$ & 109 & 307 & $\begin{array}{l}297 \\
(82 * *)\end{array}$ & 360 & 81 & 703 & $\left(\begin{array}{ll}1 & 857 \\
(1 & 642 * *)\end{array}\right.$ \\
\hline 3 & $\begin{array}{l}\text { moyenne de personnes } \\
\text { par habitation }\end{array}$ & 7.2 & 7 & 11 & 8.8 & 10.1 & 10.4 & 9 \\
\hline $\begin{array}{l}4 \\
5\end{array}$ & $\begin{array}{l}\text { nombre de ménages } \\
\text { nombre d'enfants en famille }\end{array}$ & 19 & 41 & $7 *$ & 45 & 18 & 87 & 217 \\
\hline & $\begin{array}{l}\text { a) travaillant au chantier } \\
\text { b) ne travaillant pas }\end{array}$ & $\begin{array}{r}3 \\
47\end{array}$ & $\begin{array}{r}5 \\
77\end{array}$ & $\begin{array}{l}2^{*} \\
8^{*}\end{array}$ & $\begin{array}{l}10 \\
95\end{array}$ & $\begin{array}{r}6 \\
33\end{array}$ & $\begin{array}{r}30 \\
239\end{array}$ & $\begin{array}{r}54 \\
499\end{array}$ \\
\hline 6 & $\begin{array}{l}\text { nombre d'ouvriers céliba- } \\
\text { taires }\end{array}$ & 22 & 123 & $33^{*}$ & 151 & 5 & 207 & 541 \\
\hline 7 & $\begin{array}{l}\text { nombre d'ouvriers mariés } \\
\text { loin de leur famille }\end{array}$ & 一 & 17 & 16 & 14 & 1 & 44 & 92 \\
\hline
\end{tabular}

* renseignements incomplets

** nombre de personnes pour lesquels on possède les indications complètes de recensement 
TABLEAU III

Âge des ouvriers non-qualifiés

\begin{tabular}{|c|c|c|c|c|c|c|c|c|c|}
\hline no & âges & Melbourne & Shipton & Windsor* & Brompton & Orford & Sherbrooke & TOTAL & $\%$ \\
\hline 1 & moins de 16 ans & 3 & 14 & 6 & 4 & 6 & 27 & 60 & 7 \\
\hline 2 & de 16 à 20 ans & 3 & 18 & 10 & 26 & 5 & 63 & 125 & 15 \\
\hline 3 & de 21 à 25 ans & 11 & 34 & 8 & 49 & 3 & 82 & 187 & 23 \\
\hline 4 & de 26 à 35 ans & 9 & 50 & 7 & 72 & 7 & 94 & 239 & 29 \\
\hline 5 & de 36 à 45 ans & 9 & 30 & 12 & 36 & 6 & 47 & 140 & 17 \\
\hline 6 & de 46 à 55 ans & 5 & 9 & 5 & 11 & 3 & 19 & 52 & 6 \\
\hline 7 & de 56 à 65 ans & 1 & - & 2 & - & 3 & 5 & 11 & 1 \\
\hline 8 & plus de 65 ans & - & - & - & - & 2 & 1 & 3 & - \\
\hline 9 & âge non mentionné & - & - & - & 1 & 1 & - & 2 & - \\
\hline 10 & TOTAL & 41 & 155 & 50 & 199 & 36 & 338 & 819 & \\
\hline
\end{tabular}

* incomplet 
Comme dans les autres milieux de travail de l'époque, des adolescents et même des enfants se retrouvent occupés à l'activité du chantier. Un relevé fait sur les données du recensement indique que pour chaque âge de douze à dix-sept ans, une majorité de jeunes garçons travaillent au chantier (tableau IV). À partir de 15 ans, c'est la règle générale ( $90 \%$ des cas). En ce qui concerne les plus jeunes, on trouve $46 \%$ des enfants de 11 ans au travail ; on peut même relever le cas de 3 enfants de 10 ans. Sur les 132 adolescents ou enfants entre 11 et 17 ans recensés, 54 seulement (soit $41 \%$ ) vivent dans leur famille; les autres, parfois à 12 ans déjà, vivent dans les chambrées avec des ouvriers de tous âges.

\section{TABLEAU IV}

Pourcentage des enfants \& adolescents au travail

\begin{tabular}{ccccccccc}
\hline âges (années) & 11 & 12 & 13 & 14 & 15 & 16 & 17 & TOTAL \\
\hline cas recensés & 13 & 17 & 23 & 16 & 25 & 23 & 15 & 132 \\
\hline$\%$ au travail & 46 & 65 & 61 & 69 & 88 & 96 & 87 & 75 \\
\hline
\end{tabular}

\section{4- Origines ethniques}

D'intéressantes données ressortent de l'examen du pays de naissance indiqué pour chaque personne dans le recensement. Le tableau $\mathrm{V}$ illustre la provenance des travailleurs non-qualifiés, dont l'immense majorité vient d'Irlande $(81 \%)$; ce sont d'ailleurs des Irlandais catholiques pour la plupart (75\%).

La plupart des Irlandais sont arrivés au Canada lors de la grande vague d'immigration de 1847-1849, comme on peut le déduire par l'âge et le lieu de naissance de leurs enfants: dans la plupart de ces familles en 1852, les enfants nés au Canada avaient moins de 4 ans. Les Écossais forment un contingent minime (8\%) alors que les autres nationalités sont peu importantes. On trouve peu de Canadiens français dans ce monde des chantiers ferroviaires. Plusieurs raisons ont été avancées pour expliquer cette absence. Ils auraient été impropres aux travaux pénibles du terrassement et du ballastage à cause de leur mauvaise alimentation et leur rendement de toute façon inférieur à celui des Irlandais ${ }^{19}$. On a également mis en cause leur

19 A. HELPS, op. cit., 197. 
TABLEAU V

Pays d'origine des travailleurs non-qualifiés

\begin{tabular}{l|c|c}
\hline \multicolumn{1}{c|}{ pays } & nombre & $\%$ \\
\hline Irlande (catholiques) & 621 & 75 \\
Irlande (protestants) & 53 & 61 \\
\hline Irlande (total) & 674 & 8 \\
\hline Écosse & 63 & 4 \\
Angleterre & 30 & 2 \\
Canada (britanniques) & 20 & 4 \\
Canada (c.-français) & 36 & 0.5 \\
États-Unis & 1 & 0.1 \\
Allemagne & 828 & 99.6 \\
\hline Total recensé & & \\
\hline
\end{tabular}

caractère peu docile à la discipline exigeante des chantiers ferroviaires ou la désapprobation du clergé catholique pour un métier nomade, sans que ces affirmations soient bien prouvées ${ }^{20}$. Quoi qu'il en soit, la rivalité qui existait entre eux et les Irlandais, de même que leur passivité face aux caprices des employeurs, pouvaient en faire une main-d'œuvre d'un appoint utile, en certaines circonstances du moins ${ }^{21}$.

Il est intéressant de constater que le facteur ethnique joue dans la constitution des populations de chaque «camp», particulièrement pour les non-Irlandais. Ainsi les Écossais ou les Canadiens français logent généralement chez des compatriotes. Le phénomène se constate aussi pour les croyants de dénominations religieuses minoritaires

20 G.R. STEVENS, Sixty years, op. cit., 260.

21 En 1850, lors de la construction du chemin de fer «Ogdensburg et Lac Champlain», l'ingénieur Walter Shanly pouvait écrire: "I have a strong faith in getting up a good gang of French carters who will render us independant of those Irish ruffians", et encore: "There is not the least necessity for promising Frenchmen weekly payments (...) You can make these contented with very little. Give them all they require to eat and drink. I'll warrant they'll wait for this pay ten times the patience of these d...d Irish (Shanly, op. cit., 152, 155). 
(Irlandais épiscopaliens, membres de la Free Chruch of Scotland, par exemple). Chaque habitation forme donc une unité sociale souvent bien caractéristique sur le plan ethnique ou religieux. Le voisinage semble une donnée importante de la vie quotidienne, car on voit souvent regroupés en petits quartiers des camps tenus par des ouvriers de nationalité ou de religion identiques ${ }^{22}$.

\section{5- Conditions de logement et d'alimentation}

Les cahiers des recenseurs indiquent la répartition précise par unité d'habitation des personnes interrogées ainsi que des détails sur le type de construction des logements; ils permettent ainsi d'évaluer la densité des populations des chantiers ainsi que les conditions de logement (Tableau II). Les maisons de charpente ou de brique ou même de style plus fruste, pièce sur pièce (log cabin), sont rares et souvent occupées par les ingénieurs ou les contremaîtres. La majorité des travailleurs demeure dans des camps (shanties), habitations provisoires qui bordent à intervalles irréguliers le tracé de la voie ${ }^{23}$. Si en moyenne un camp compte 9 personnes avec sa famille et ses pensionnaires, il n'est pas rare de rencontrer des logements de 15, 20 ou même 30 personnes. Dans le cas de camps très peuplés, on remarque la présence de jeunes filles ou de veuves qui aident la mère de famille à tenir ces vastes maisonnées de chambreurs; la plupart de ces domestiques sont d'origine irlandaise. Faute d'un tel personnel, plusieurs familles parviennent à tenir pension grâce aux filles aînées qui remplissent le rôle d'aides-ménagères. Les chantiers du St. Lawrence and Atlantic diffèrent ainsi des chantiers forestiers: le service alimentaire et l'hébergement ne sont pas fournis par l'entrepreneur, mais par des ouvriers aux autres ouvriers. La base de cet état de choses est évidemment la disponibilité de la main-d'œuvre féminine quasi-gratuite fournie principalement par les structures familiales. Par ailleurs, les entrepreneurs répugnent à engager leurs hommes «pension comprise», ce système leur revenant trop cher et

22 Exemples: Sherbrooke, shanties no 9, 10, 12, 13 et 14 (un ménage canadien-français protestant et quatre irlandais épiscopaliens créent un îlot dans une mer de shanties irlandais-catholiques); shanties 33 à 41 (épiscopaliens et fidèles de la Free Church).

23 Généralement hauts de 8 pieds et larges de 12, ces camps ont une longueur variable. Le toit est goudronné. Voir Journal of Leg. Ass. of the Prov. Of Canada, 13 (1854-55): app. $\operatorname{EEEE~(n.p.)~}$ 
leur causant trop d'ennuis et vont même jusqu'à payer des gages plus élevés aux ouvriers, pourvu que ceux-ci se débrouillent eux-mêmes:

(...) if I could get some persons to undertake to board the men, I would give up doing it at once. I would give the use of the Shanty of course and even add a bonus to anyone undertaking it (...) If you can't find someone to take the shanty, of course we are in for it, but it would save a great deal to pay every man double wages and have nothing to do with feeding him, ${ }^{24}$

Your Shanty charges are big (...) Board the men we will not, and until you can get someone to take the Shanty, you may give high wages to the men and let them do their own purveying ${ }^{25}$.

Signalons enfin que beaucoup de ces habitations sont flanquées d'écuries; on peut au moins le constater pour la zone de Sherbrooke où le recenseur consciencieux a intégré dans le questionnaire agricole les ouvriers du chantier. Les chevaux, qui aident aux opérations de charroi ou de déblaiement, sont l'apanage non seulement des entrepreneurs ou des contremaîtres, mais aussi de 25 ouvriers qui possèdent leur couple d'animaux. Quelques très rares ménages de travailleurs élèvent une vache ou un cochon.

\section{6- Catégories professionnelles}

Ainsi que le montre le tableau VI, environ un millier de personnes exercent un métier rémunéré dans les chantiers ferroviaires de la région Richmond-Sherbrooke.

De ce nombre, près de $90 \%$ sont des journaliers (laborers) et constituent la masse des ouvriers non-qualifiés. Les artisans sont peu nombreux (3\%) et se recrutent souvent parmi les forgerons, charpentiers, cordonniers ou maçons. Le personnel technique ou de cadre représente environ $4 \%$. Ces deux dernières catégories sont l'apanage de groupes ethniques bien particuliers (voir Tableau VII).

Les Canadiens ne représentent qu'une faible minorité de ces catégories professionnelles qualifiées tandis que les tâches exigeant des qualifications précises (artisans, ingénieurs) sont souvent le fait d'Écossais; des postes plus pratiques comme contremaîtres sont oc-

24 Shanly, op. cit., 137.

${ }_{25}$ Ibid., 141. L'ameublement est aussi à la charge des ouvriers: "we will fit up the House properly, but not provide an atom of furniture of any kind" (ibid., 144). 
TABLEAU VI

Catégories professionnelles des travailleurs des chantiers

\begin{tabular}{|c|c|c|c|c|c|c|c|c|c|}
\hline no & & & Melbourne & Shipton & Windsor & Brompton & Orford & Sherbrooke & TOTAL \\
\hline 1 & nombre & de journaliers & 41 & 155 & $129 *$ & 199 & 36 & 338 & 898 \\
\hline 2 & " & d'artisans & - & 8 & 5 & 2 & 1 & 10 & 27 \\
\hline 3 & $"$ & d'employés & - & 1 & - & 2 & - & 1 & 4 \\
\hline 4 & $"$ & de contremaîtres & - & 1 & 4 & - & - & 9 & 14 \\
\hline 5 & $"$ & d'ingénieurs & - & 2 & 1 & - & - & 2 & 5 \\
\hline 6 & $"$ & d'entrepreneurs & 1 & 5 & 5 & 3 & - & 6 & 20 \\
\hline 7 & $"$ & de commercants & - & - & - & - & - & 2 & 2 \\
\hline 8 & $"$ & de domestiques & - & 16 & - & 6 & - & 12 & 34 \\
\hline 9 & Totaux & & 43 & 188 & 144 & 212 & 37 & 380 & 1004 \\
\hline
\end{tabular}

* incomplet 
TABLEAU VII

Pays d'origine des cadres et travailleurs qualifiés

\begin{tabular}{l|c|c|c|c|c}
\hline & artisans & contremaitres & entrepreneurs & ingénieurs & TOTAL \\
\hline Écosse & 10 & - & 5 & 2 & 17 \\
Angleterre & 1 & 3 & 4 & - & 8 \\
Irlande (cath.) & 5 & 7 & 4 & - & 16 \\
Irlande (prot.) & 5 & $\frac{1}{3}$ & $\frac{5}{7}$ & $\frac{1}{1}$ & 5 \\
États-Unis & - & - & 1 & $\frac{1}{8}$ & 4 \\
Canada (ang.) & 3 & - & - & 1 & 1 \\
Canada (fr.) & 3 & - & - & 5 & 66 \\
Pologne & - & - & 20 & & \\
\hline TOTAL & 27 & 14 & &
\end{tabular}

cupés fréquemment par des Irlandais alors que la responsabilité générales des chantiers est aux mains d'entrepreneurs originaires des pays détenant une expérience technologique avancée dans la construction ferroviaire (Écosse, Angleterre, États-Unis). Les différences professionnelles n'apparaissent guère par les logements: les artisans partagent les camps avec les travailleurs non-qualifiés; contremaîtres et entrepreneurs logent assez souvent dans de telles demeures de fortune, mais il est vrai que lorsque l'occasion se présente, on les voit généralement louer des maisons de charpente ou de brique le long de la voie. Les ingénieurs mènent par contre un train de vie plus cossu, au milieu d'une nombreuse domesticité. ${ }^{26}$ Le taux de scolarité des enfants varie aussi selon la profession du père. Dans les secteurs ruraux de la voie, les enfants des ouvriers non-qualifiés ne fréquentent guère l'école alors que ceux des cultivateurs voisins le font; les enfants des travailleurs qualifiés constituent de ce fait plus de $75 \%$ des enfants entre 7 et 11 ans qui sont scolarisés et la totalité de ceux entre 12 et 14 ans.

26 Casimir Gzowski, ingénieur-en-chef de la ligne, associé d'Alexander Galt, réside dans le quartier nord de Sherbrooke, dans une belle demeure en briques, voisin d'avocats et de hauts fonctionnaires (RC, Sherbrooke dans Orford, 5). 


\section{7- Salaires et conditions de travail.}

Si les recensements sont muets sur ce point, les journaux de l'époque, les documents officiels et les correspondances des ingénieurs nous fournissent un bon nombre de précisions sur les conditions de travail. Il ressort du tableau VIII que les ouvriers non-qualifiés d'un chantier ferroviaire en 1852 étaient payés au moins 80 cents par jour, mais que les salaires pouvaient varier en fonction du chantier et de la saison.

\section{TABLEAU VIII}

Salaires quotidiens des travailleurs des chantiers (chemins de fer et canaux) (en dollars), 1850-1853

\begin{tabular}{|c|c|c|c|c|c|}
\hline année & $\begin{array}{l}\text { Hiver } \\
1850^{27}\end{array}$ & $\begin{array}{l}\text { Hiver } \\
1852^{28}\end{array}$ & $\begin{array}{c}\text { Été } \\
1852^{29}\end{array}$ & $\begin{array}{l}\text { Été } \\
1852^{28}\end{array}$ & $\begin{array}{l}\text { Hiver } \\
1853^{28}\end{array}$ \\
\hline journalier & 0.75 & 0.60 & $\begin{array}{l}0.80 \text { à } \\
1.00\end{array}$ & $\begin{array}{l}0.70 \text { à } \\
0.80\end{array}$ & 0.60 \\
\hline maçon & - & - & $\begin{array}{l}1.50 \text { à } \\
2.00\end{array}$ & - & - \\
\hline charpentier & - & - & $\begin{array}{l}1.30 \mathrm{à} \\
2.00\end{array}$ & - & - \\
\hline cuisinier & 0.80 & - & - & - & - \\
\hline contremaître & $\begin{array}{l}1.00 \text { à } \\
2.60\end{array}$ & - & - & - & - \\
\hline $\begin{array}{l}\text { charretier } \\
\text { (avec attelage) }\end{array}$ & 2.00 & - & - & - & - \\
\hline
\end{tabular}

Les salaires relativement élevés payés aux artisans s'expliquent sans doute par la nécessité d'attirer au Canada des ouvriers qualifiés d'Écosse ou d'Angleterre.

27 Chantier du chemin de fer «Ogdensburg et Lac Champlain» (cf. Shanly, op. cit., $130 ; 140$ ).

${ }_{28}$ Chantier de canaux (Ontario) (cf. Journal of Leg. Ass. of the Prov. of Canada, loc. cit.)

29 Chantiers du Grand-Tronc (cf. A. HELPS, op. cit., app. C; A.W. CURRIE, op. cit., 29). 
Le rythme d'avancement de la voie ralentit certes durant les mois d'hiver, mais à la bonne saison, les travaux sont poussés vigoureusement par les entrepreneurs, les travailleurs se divisant en certains cas en une équipe de jour et une de nuit ${ }^{30}$. Une cloche ponctue le rythme quotidien du chantier ${ }^{31}$. La fébrilité qui envahit les chantiers et pousse les travailleurs à faire avancer la voie toujours plus vite, vient de loin; contremaîtres, sous-traitants, entrepreneurs ne font que relayer la pression des milieux d'affaires et des actionnaires soucieux de voir l'entreprise rapporter au plus tôt d'intéressants profits. Pour le travailleur de la voie cependant, cette hâte se traduit par une augmentation des risques d'accidents, les contremaitres accroissant les cadences de travail et faisant peu de cas des exigences de sécurité prévues par les ingénieurs. Aussi les accidents mortels sont-ils nombreux ${ }^{32}$. Aucune enquête du coroner ne suit généralement ces décès, ce qui n'incite guère les entrepreneurs à faire des efforts pour limiter les risques ${ }^{33}$. Quant à la qualité de la construction de la voie ferrée, elle ne pouvait que se ressentir de la négligence instaurée en système par les entrepreneurs et leurs sous-traitants. Le chemin de fer du St. Lawrence and Atlantic passa bientôt pour une des lignes les plus mal construites de l'Amérique du Nord ${ }^{34}$ et la compagnie du Grand-Tronc qui le racheta bientôt dut dépenser un million de dollars entre Montréal et Portland pour le réparer ${ }^{35}$.

30 Shanly, op. cit., 151, 153, 154.

31 Sherbrooke Gazette cité par Stanstead Journal, 3 mars 1853.

32 Un sondage dans les journaux de la région entre mars et août 1852 révèle les accidents suivants: un ouvrier mort enseveli dans un éboulement près de Sherbrooke (Stanstead Journal, 4 mars 1852); un ouvrier tué par la chute d'une poutre au pont de Lennoxville, (ibid., 10 juin 1852); un ouvrier blessé en tombant du pont de Brompton (ibid.) ; un jeune ouvrier mort noyé, en tombant du pont de Lennoxville (Sherbrooke Gazette citée par Montreal Gazette, 17 juin 1852); un charpentier tombe du pont à Richmond et se noie (Montreal Gazette, 18 août 1852); deux tués et quatre blessés dans des éboulements entre Lennoxville et Compton (Sherbrooke Gazette cité par Stanstead Journal, 19 août 1852).

33 Stanstead Journal, 19 août 1852.

34 «It is doubtful that with their spongy roadbeds, abrupt gradients, jughandle curves, there were worse railways anywhere on the continent» (B.R. STEVENS, op. cit., 264). Voir aussi A.W. CURRIE, op. cit., 56 et T.S. BROWN, op. cit., 14. Les spécifications requises par la compagnie étaient pourtant très exigeantes comme on peut le voir dans A.C. MORTON, Report on the gauge for the St. Lawrence and Atlantic Railroad, (Montréal, Canada Gazette Office, 1847), 69-79.

35 G.R. STEVENS, op. cit., 265; T.C. KEEFER, "The Early history of the Grand Trunk Railway» in Canada. An Encyclopedia of the Country, J.C. HOPKINS, ed., (6 vols, Toronto, 1898-1900), 2: 97-103. 


\section{8- Alcool et violence}

La présence d'une importante population ouvrière, sans attache avec les habitants de la région et relativement bien payée, pousse de nombreux marchands d'alcool à installer à proximité des chantiers des débits de boisson. L'ivrognerie, les bagarres, les violences deviennent dès lors monnaie courante le long de la voie et dans les villes et villages avoisinants. La presse locale s'émeut régulièrement, non de ces phénomènes en soi, mais de leurs effets sur les populations sédentaires ${ }^{36}$ et demande aux autorités municipales de restaurer l'ordre. Faute de forces policières suffisantes, celles-ci se sentent incapables de faire appliquer les lois sur la vente d'alcool ou sur le maintien de la paix. À Sherbrooke, où la consommation d'alcool aurait quadruplé, où les rixes dans les rues seraient devenues fréquentes et la dynamite sans cesse véhiculée dans les rues ${ }^{37}$, des citoyens déclenchent un mouvement pour détacher la ville de la municipalité de comté, jugée inefficace, et demander son incorporation en municipalité séparée ${ }^{38}$. Le St. Lawrence and Atlantic ne jouit guère de l'estime des populations de la région, principalement des propriétaires, mécontents de devoir payer de leur poche l'entretien de policiers que la compagnie aurait dû, selon eux, engager pour empêcher la vente d'alcool ou maintenir l'ordre ${ }^{39}$.

Les autorités judiciaires du District de St-François s'émeuvent peu, il faut bien l'admettre, devant les violences qui deviennent monnaie courante. Il faut attendre les événements de Coaticook de février 1853 pour voir l'appareil répressif se mettre en branle. L'affaire commence le 22 janvier, quand deux policiers envoyés à un chantier entre Compton et Coaticook pour procéder à l'arrestation d'ouvriers accusés de violence, sont repoussés par les travailleurs et obligés de rebrousser chemin sans pouvoir exécuter leur mandat. Le 15 février, le shérif du district G.F. Bowen, en personne, et trois poli-

\footnotetext{
19 mai 1853.

37 Sherbrooke Gazette, 8 novembre 1851.

38 Incorporation sanctionnée le 28 juin 1852.

39 Stanstead Journal, $1^{\text {er }}$ janvier 1852; 19 mai 1853. L'inauguration du tronçon Montréal-Sherbrooke en septembre 1852 donne par contre aux honorables membres du barreau, juges, journalistes, députés, sous la présidence du gouverneur Elgin luimême, l'occasion de participer à un banquet à la gare de Sherbrooke. Plus de 500 bouteilles de champagne furent vidées aux frais de la Compagnie. L'ivresse fut générale ce jour-là et les rues de Sherbrooke peu sûres. Cf. les relations détaillées dans Montreal Gazette, 14 septembre 1852 et dans J. VANSITTART, op. cit., 137-138.
}

36 Sherbrooke Gazette, 2 août 1851; Stanstead Journal, $1^{\text {er }}$ janvier 1852; 
ciers, venus arrêter les réfractaires, sont attaqués par les ouvriers irlandais, battus violemment et laissés pour morts ${ }^{40}$. La nouvelle de cet attentat sur la personne du shérif sème la panique parmi les autorités qui mobilisent la milice locale et demandent au gouverneur un détachement de l'armée. Le 24 février, c'est une troupe de 150 soldats et miliciens qui surprend à l'aube le chantier de Coaticook, capture plusieurs ouvriers qui sont ramenés à la prison de Sherbrooke. Au total, 35 travailleurs furent incarcérés ${ }^{41}$. L'opération est complétée à la fin du mois de mars par une descente conjointe des polices de Sherbrooke et du Vermont dans les chantiers situés aux environs de la frontière des États-Unis. Quelques ouvriers impliqués dans l'attaque du shérif sont arrêtés de même qu'une vingtaine de vendeurs d'alcool installés le long de la ligne; les stocks de boisson sont systématiquement détruits ${ }^{42}$. Cette intervention énergique des forces de l'ordre fut sans doute efficace, mais bien tardive. La construction du St. Lawrence and Atlantic achevait. Depuis février, les trains reliaient quotidiennement Portland à Island Pond (Vermont) ${ }^{43}$; en juillet 1853, le dernier tronçon entre Island Pond et Coaticook était terminé et le premier train de Montréal à Portland l'empruntait ${ }^{44}$. Les travailleurs, ces hommes « of a low type of character $»^{45}$ quittaient la région pour d'autres chantiers et les citoyens du District de SaintFrançois pouvaient maintenant jouir en toute tranquillité des bienfaits de l'ère de la vapeur.

\section{Conclusions}

1- L'intérêt des recensements nominaux de 1851-52 pour la région de Sherbrooke est donc de nous offrir une base statistique sérieuse sur le phénomène des ouvriers des chantiers ferroviaires. Ils éclairent les conditions de vie, les modes d'existence et les caractéristiques sociales d'un type de travailleurs migrants, généralement mal saisi par les sources.

40 Sous le titre "Outrage on the Railroad line», le Stanstead Journal du 24 février 1853 donne un récit détaillé des événements. George Frederick Bowen, en plus d'être shérif du district, est le premier maire de la nouvelle municipalité de la ville de Sherbrooke.

41 Sherbrooke Gazette cité par Stanstead Journal, 3 mars 1853.

42 Stanstead Journal, 7 avril 1853.

43 Ibid., 17 février 1853.

44 Ibid., 18 juillet 1853.

45 Pour reprendre l'expression du Stanstead Journal, 19 mai 1853. 
2- Des données essentielles de la vie et, pourrait-on ajouter, de la mort du chantier échappent à l'enquête des recenseurs: les salaires, les accidents de travail, la violence, l'alcoolisme... Ces données sont davantage, bien qu'imparfaitement, évoquées par les journaux locaux, qui reflètent le point de vue des populations autochtones, particulièrement des possédants. Avec d'autres sources contemporaines, comme les mémoires des ingénieurs, les journaux nous permettent de saisir davantage l'impact sur une région du passage des ouvriers des chantiers.

3- Il serait bon de tenir compte désormais de l'effet du phénomène des travailleurs migrants dans les décomptes de population des régions traversées par des chemins de fer. Dans le cas étudié, la population autochtone de la région Richmond-Sherbrooke a été en 1851 gonflée de quelque $23 \%$ par la présence, durant les semaines du recensement, de 1800 personnes liées aux chantiers. Celles-ci, pour la plupart, ont suivi les progrès de la voie vers les États-Unis et ont quitté la région. Il en résulte aussi de fausses impressions sur la croissance de la population de la région entre 1844 et 1861 .

4- Il serait intéressant par ailleurs d'effectuer une comparaison avec les caractères et les conditions de la classe ouvrière vivant à la même époque à Sherbrooke, dans les manufactures textiles par exemple. Cette étude, actuellement en cours, peut également trouver sa base documentaire dans les recensements nominaux.

5- Enfin, il y aurait lieu de procéder à des sondages dans les recensements manuscrits de 1851,1861 ou 1871 pour rechercher s'il n'existerait pas dans ces documents de traces d'autres chantiers de construction ferroviaire au Canada. On peut penser par exemple au chemin de fer Prescott and Bytown en 1851 ou à l'Intercolonial en 1871. De telles études permettraient entre autres de suivre l'évolution des conditions de travail et des traits sociaux des travailleurs entre les deux grands moments de l'histoire ferroviaire canadienne au XIX siècle, celle du Grand-Tronc vers 1850 et celle du Canadien Pacifique, trente ans plus tard. 